\title{
JOINT BROWDER SPECTRA AND TENSOR PRODUCTS
}

\author{
A.T. DASH
}

\begin{abstract}
There exists in the literature several notions of joint spectra which can be generalized to joint Browder spectra. The purpose of this note is to show that various notions of joint Browder spectra coincide for a special class of operators.
\end{abstract}

We first consider various notions of joint spectra existing in the literature (see, for example, [6], [7] and [14]).

DEFINITION 1 . Let $A=\left(A_{1}, \ldots, A_{n}\right)$ be an $n$-tuple of commuting operators (bounded linear transformations) on a complex Banach space $X$.

(a) The joint spectrom $\sigma(A)$ of $A$ is defined as

$$
\sigma(A)=\sigma^{2}(A) \cup \sigma^{r}(A),
$$

where the left (right) joint spectrum $\sigma^{2}(A)\left(\sigma^{r}(A)\right)$ is defined as the set of all points $z=\left(z_{1}, \ldots, z_{n}\right)$ in $\mathbf{c}^{n}$ (the $n$-fold Cartesian product of the set of all complex numbers $\mathbb{C}$ ) such that $\left\{A_{j}-z_{j}\right\}_{1 \leq j \leq n}$ generates a proper left (right) ideal in the algebra $B(X)$ of all operators on $X$.

(b) The commutant joint spectrm $\sigma^{l}(A)$ is defined as the set of all

Received 7 January 1985. The research was supported by the NSERC Grant A7545.

Copyright Clearance Centre, Inc. Serial-fee code: 0004-9727/85 $\$$ A2.00 +0.00 . 
$z=\left(z_{1}, \ldots, z_{n}\right)$ in $\mathbb{C}^{n}$ such that the set $\left\{A_{j}-z_{j}\right\}_{1 \leq j \leq n}$ is contained in a proper (two sided) ideal of $A^{\prime}$, where $A^{\prime}$ is the set of all elements of $B(X)$ that commute with $A_{1}, \ldots, A_{n}$.

(c) The double commutant spectrum $\sigma^{2}(A)$ of $A$ is the set of all $z=\left(z_{1}, \ldots, z_{n}\right)$ in $\mathbb{C}^{n}$ such that the closed ideal generated by the set $\left\{A_{j}{ }^{-z}\right\}_{1 \leq j \leq n}$ is a proper ideal in $A^{\prime \prime}$, where $A^{\prime \prime}=\left(A^{\prime}\right)^{\prime}$.

(d) The Tayzor joint spectrum $\sigma^{T}(A)$ of $A$ is defined to be the set of all complex $n$-tuple $z=\left(z_{1}, \ldots, z_{n}\right)$ for which $A-z=\left(A_{1}-z_{1}, \ldots, A_{n}-z_{n}\right)$ is singular.

The n-tuple $A$ is said to be non-singular if the Koszul complex $E(X, A)=\left\{E_{k}^{n}(X), d_{k}^{n}\right\}_{k \in J} \quad$ is exact, where $J$ is the set of all integers, $E^{n}$ is the complex algebra with identity $e$, generated by indeterminants $e_{1}, \ldots, e_{n}, E_{k}^{n}(X)=E_{k}^{n} \otimes X \quad\left(E_{k}^{n}\right.$ is generated by $e_{j_{1}} \wedge \ldots \wedge e_{j_{k}}$ with $\left.1 \leq j_{1}<\ldots<j_{k} \leq n\right)$, and $d_{k}^{n}\left(x \otimes e_{j_{1}} \wedge \ldots \wedge e_{j_{k}}\right)=\sum_{i=1}^{k}(-1)^{i+1} A_{j_{i}} x \otimes e_{j_{1}} \wedge \ldots \wedge \hat{e}_{j_{i}} \wedge \ldots \wedge e_{j_{k}}$. See $[14]$.

(e) The polynomial spectrum $\sigma^{P}(A)$ of $A$ is defined as follows: $\sigma^{P}(A)=\left\{z=\left(z_{1}, \ldots, z_{n}\right): p(z) \in \sigma(p(A))\right.$ for all polynomials $\left.p: \phi^{n}+\phi\right\}$. Consult [1] for details.

The discussions above lead to the following inclusion relation between the various kinds of spectra

$$
\sigma(A) \subseteq \sigma^{T}(A) \subseteq \sigma^{l}(A) \subseteq \sigma^{2}(A) \subseteq \sigma^{P}(A)
$$

The first containment is a direct consequence of Proposition 2.10 [15] for 
operators on a Hilbert space. The fact that $\sigma^{T}(A) \subseteq \sigma^{1}(A) \subseteq \sigma^{2}(A)$ is discussed in [14] and it is not hard to see that $\sigma^{2}(A) \subseteq \sigma^{P}(A)$. See [1].

Recall that all these notions of spectra coincide in case of a single operator. However, we will show below that there exists a special class of operators for which the notions of joint spectra discussed in (b), (c), (d) and (e) coincide. See Theorem 2 below.

Let $X_{1}, \ldots, X_{n}$ be Banach spaces, and let $B_{i}$ be an operator on $X_{i}$ for all $i, 1 \leq i \leq n$. Let $Y=X_{1} \otimes \ldots \otimes X_{n}$ be the completion of the tensor product with respect to some cross-norm. Then

$$
\begin{aligned}
& A_{1}=B_{1} \otimes I_{2} \otimes \ldots \otimes I_{n}, A_{2}=I_{1} \otimes B_{2} \otimes I_{3} \otimes \ldots \otimes I_{n}, \ldots, \\
& A_{k}=I_{1} \otimes \ldots \otimes B_{k} \otimes I_{k+1} \otimes \ldots \otimes I_{n}, \ldots, A_{n}=I_{1} \otimes \ldots \otimes B_{n}
\end{aligned}
$$

are commuting operators on $Y$. It is proved in [10] that

$$
\sigma^{2}\left(A_{1}, \ldots, A_{n}\right)=\prod_{k=1}^{n} \sigma\left(B_{k}\right)
$$

Recently Ceausescu and Vasilescu [5] proved that (1) holds for the Taylor joint spectrum (that is, $\sigma^{T}\left(A_{1}, \ldots, A_{n}\right)=\prod_{k=1}^{n} \sigma\left(B_{k}\right)$ ) in the case that the $X_{i}$ 's are complex Hilbert spaces. Combining all these results one concludes easily that the Taylor joint spectrum, the commutant joint spectrum, the double commutant joint spectrum and the polynomial joint spectrum all coincide for these classes of operators. Thus we have explicitly the following

THEOREM 2. Let $A=\left(A_{1}, \ldots, A_{n}\right)$ be the n-tuple of operators $A_{k}=I_{1} \otimes \ldots \otimes I_{k-1} \otimes B_{k} \otimes I_{k+1} \otimes \ldots \otimes I_{n}, k=1, \ldots, n$, on the Hilbert space $H=H_{1} \otimes \ldots \otimes H_{n}$ as described above. Then

$$
j(A)=\prod_{k=1}^{n} \sigma(B)
$$

for $j=1,2, P, T$.

Proof. The result follows easily from the following observation and 
the fact that $\sigma\left(A_{k}\right)=\sigma\left(B_{k}\right)$ for $k=1, \ldots, n$ (see Proposition 3)

$$
\sigma^{T}(A) \subseteq \sigma^{I}(A) \subseteq \sigma^{2}(A) \subseteq \sigma^{P}(A) \subseteq \prod_{k=1}^{n} \sigma\left(A_{k}\right)=\prod_{k=1}^{n} \sigma\left(B_{k}\right)=\sigma^{T}(A) .
$$

REMARK 1. Note that Theorem 2 is true for Banach space operators for $j=1,2, P$. However, for $j=T$, the result is still unknown for Banach spaces.

REMARK 2. It is not hard to see that Theorem 2 is not valid for the joint spectrum $\sigma(A)$ in Definition 1 (a). Indeed, consider the operators $A_{1}=U \otimes I$ and $A_{2}=I \otimes U^{*}$, where $U$ is the unilateral shift defined by Ue $e_{n}=e_{n+1}$ and $\left\{e_{n}\right\}_{0}^{\infty}$ is an orthonormal basis of a Hilbert space. Thus we have

$$
\begin{aligned}
\sigma\left(A_{1}, A_{2}\right)=\sigma^{2}\left(A_{1}, A_{2}\right) \cup \sigma^{r}\left(A_{1},\right. & \left.A_{2}\right) \\
& =(\partial D \times D) \cup(D \times \partial D) \neq D^{2}=\sigma(U) \times \sigma\left(U^{*}\right),
\end{aligned}
$$

where $D$ is the closed unit disc and $\partial D$ its boundary; see [9].

Furthermore, it is also clear that all the above notions of joint spectra coincide in case of operators in finite dimensional spaces.

PROPOSITION 3. Consider the operator $B \otimes 1$, where $B$ is an operator on a Hilbert space and 1 is the identity operator. Then

$$
\sigma_{b}(B \otimes 1)=\sigma_{e}(B \otimes 1)=\sigma_{\omega}(B \otimes 1)=\sigma(B \otimes 1)=\sigma(B),
$$

where $\sigma_{\omega}(S)$ is the Weyl spectrum of an operator $S$ defined by $\sigma_{\omega}(S)=\prod_{K} \sigma(S+K)$ and $K$ is a compact operator. For other notions of spectra see Definition 4.

Proof. From Lemma 4.2 [2] it follows that $\sigma(B \otimes 1)=\sigma(B)$ and $\sigma_{e}(B \otimes 1)=\sigma(B)$. Hence we obtain

$$
\sigma(B)=\sigma_{e}(B \otimes 1) \subseteq \sigma_{\omega}(B \otimes 1) \subseteq \sigma_{b}(B \otimes 1) \subseteq \sigma(B \otimes 1)=\sigma(B) .
$$

This proves the proposition.

However, the situation in case of a pair of operators is different (see [12] and [13]). This will be the subject of our discussion for the 
rest of the sections of this paper.

Let us first introduce various notions of joint essential spectra and joint Browder spectra.

Let $A=\left(A_{1}, \ldots, A_{n}\right)$ be an n-tuple of elements in $B(X)$. Let $A_{e}^{1}$ be the almost commutant of $A$ (commute modulo the compact operators), $A_{e}^{2}$ be the almost double commutant of $A$.

The reader is referred to [3], [12] and [13] for details.

DEFINITION $4 . \quad z=\left(z_{1}, \ldots, z_{n}\right) \in \rho_{b}^{j}(A)$ if $z \in \rho_{b}^{j}(A)$ in $A_{e}^{j}$ and there exists a deleted neighborhood $N$ of $z$ such that if $\lambda=\left(\lambda_{1}, \ldots, \lambda_{n}\right) \in N$, then $\lambda \in \rho^{j}(A)$ for $j=1,2, T$. Now we say that $z \in \sigma_{b}^{j}(A)$, the joint Browder spectrum of $A$ if $z \epsilon^{\prime} \rho_{b}^{j}$ for $j=1,2, T$. Here $\epsilon^{\prime}$ means "does not belong to". For the definition of polynomial joint Browder spectrum see [4]. Moreover, it follows from Definition 4 and Theorem 8 in [4] that

$$
\sigma_{b}^{j}(A)=\sigma_{e}^{j}(A) \cup\left\{\text { accumulation points of } \sigma^{j}(A)\right\},
$$

for $j=1,2, P, T$. Here $\sigma_{e}^{j}(A)$ is the joint essential spectrum of $A$ for $j=1,2, P, T$. See [4], [6], [8] and [12].

This together with the fact that any accumulation point of a set $G$ is also an accumulation point of a set containing $G$ and that $\sigma_{e}^{T}(A) \subseteq \sigma_{e}^{l}(A) \subseteq \sigma_{e}^{2}(A) \subseteq \sigma_{e}^{P}(A)$ imply that $\sigma_{b}^{T}(A) \subseteq \sigma_{b}^{1}(A) \subseteq \sigma_{b}^{2}(A) \subseteq \sigma_{b}^{P}(A)$.

Next we shall prove a theorem on joint Browder spectra (Theorem 7) which is analogous to Theorem 2 on joint spectra. First of all note that Schechter and Snow [12] showed that if $A_{1}=B \otimes 1$ and $A_{2}=1 \otimes C$ are operators on the Banach space $x_{1} \otimes X_{2}$, then

$$
\left\{\sigma_{e}(B) \times \sigma(C)\right\} \cup\left\{\sigma(B) \times \sigma_{e}(C)\right\} \cong \sigma_{e}^{2}\left(A_{1}, A_{2}\right) .
$$

We prove analogous results for various notions of joint essential spectra of operators on Hilbert spaces as given in Theorem 6. This generalizes the 
results of Schechter and Snow to various notions of joint essential spectra, in particular to Taylor joint essential spectra which is a smaller set than that of the double commutant joint essential spectra, for tensor products of operators on Hilbert spaces.

LEMMA 5. If $B$ and $C$ are operators on Hilbert spaces $H_{1}$ and $H_{2}$, then

$$
\left\{\sigma_{e}^{2}(B) \times \sigma(C)\right\} \cup\left\{\sigma(B) \times \sigma_{e}^{2}(C)\right\} \subseteq \sigma_{e}^{j}(A) \text { for } j=1,2, P, T,
$$

where $A=\left(A_{1}, A_{2}\right)$ and $A_{1}=B \otimes 1$ and $A_{2}=1 \otimes C$ as defined above. Here $\sigma_{e}^{2}(T)$ denotes the left joint essential spectrum of an operator $T$ (see $[8]$ ).

Proof. It is enough to show that $\sigma_{e}^{Z}(B) \times \sigma(C) \subseteq \sigma_{e}^{T}(A)$. In other words it is sufficient to show that if $0 \in \sigma_{e}^{l}(B)$ and $0 \in \sigma(C)$, then $(0,0) \in \sigma_{e}^{T}(A)$. If $0 \in \sigma_{e}^{2}(B)$ and $0 \in \sigma(C)$, then there exists an orthonormal sequence $\left\{e_{n}\right\}$ [11] and a sequence $\left\{x_{n}\right\}$ of unit vectors such that $\left\|B e_{n}\right\| \rightarrow 0$, and either $\left\|C x_{n}\right\| \rightarrow 0$ or $\left\|C^{*} x_{n}\right\| \rightarrow 0$. Set $z_{k}=e_{k} \otimes x_{k}$ and

$$
\zeta_{k}=\left(\begin{array}{c}
z_{k} \\
0
\end{array}\right) \text { if }\left\|C^{*} x_{k}\right\| \rightarrow 0
$$

and

$$
\zeta_{k}^{\prime}=\left(\begin{array}{c}
0 \\
z_{k}
\end{array}\right) \text { if } \quad\left\|C x_{k}\right\| \rightarrow 0 .
$$

Clearly the sequences $\zeta_{k}$ and $\zeta_{k}^{\prime}$ are orthonormal and hence weakly tend to zero. Furthermore, we know that $A$ is Fredholm if and only if the matrix

$$
V=\left(\begin{array}{cc}
A_{1} & A_{2} \\
-A_{2}^{*} & A_{1}^{*}
\end{array}\right)
$$

is Fredholm. This fact together with the definitions of $\zeta_{\vec{k}}$ and $\zeta_{\vec{k}}^{\prime}$ 
implies that either $\left\|V \zeta_{k}\right\| \rightarrow 0$ or $\left\|V^{*} \zeta_{k}^{\prime}\right\| \rightarrow 0$. This means $V$ is not Fredholm [11] and hence $A$ is not Fredholm. Therefore, $(0,0) \in \sigma_{e}^{T}(A)$. Since $\sigma_{e}^{T}(A) \subseteq \sigma_{e}^{1}(A) \subseteq \sigma_{e}^{2}(A) \subseteq \sigma_{e}^{P}(A)$, the result is proved.

THEOREM 6. If $B, C$ and $A$ are as given in Lemma 5, then

$$
\left\{\sigma_{e}(B) \times \sigma(C)\right\} \cup\left\{\sigma(B) \times \sigma_{e}(C)\right\} \subseteq \sigma_{e}^{j}(A) \text { for } j=1,2, P, T .
$$

Proof. By similar techniques as given in the above lemma one can show that

$$
\left\{\sigma_{e}^{r}(B) \times \sigma(C)\right\} \cup\left\{\sigma(B) \times \sigma_{e}^{r}(C)\right\} \subseteq \sigma_{e}^{j}(A) \text { for } j=1,2, P, T .
$$

This fact together with Lemma 5 proves the results. Here $\sigma_{e}^{r}(T)$ denotes the right joint essential spectrum of an operator $T$ (see [8]).

The following theorem was proved by Snow [13] for $\sigma_{b}^{2}(A)$, where $A=\left(A_{1}, A_{2}\right), A_{1}=B \otimes 1, A_{2}=1 \otimes C$ and $B, C$ are operators on Banach spaces $X_{1}$ and $X_{2}$. However, we show below that various notions of joint Browder spectrum coincide for operators $A_{1}$ and $A_{2}$ on Hilbert space $H_{1} \otimes H_{2}$. This is in some sense a generalization of Snow's result to various notions of joint Browder spectrum for tensor products of operators on Hilbert spaces.

THEOREM 7. If $A_{1}, A_{2}$ and $A$ are as defined in Lemma 5, then (2) $\sigma_{b}^{j}(A)=\left\{\sigma_{b}(B) \times \sigma(C)\right\} \cup\left\{\sigma(B) \times \sigma_{b}(C)\right\}$ for $j=1,2$ and $T$.

Proof. We first show the equality of (2) for Taylor joint spectrum $\sigma^{T}(A)$. From Theorem 2 we have $\sigma^{T}(A)=\sigma(B) \times \sigma(C)$. Let $z_{1} \epsilon \sigma_{b}(A)$ and $z_{2} \in \sigma(C)$. If $z_{1} \in \sigma_{e}(B)$, then it follows from Theorem 6 that $\left(z_{1}, z_{2}\right) \in \sigma_{e}^{T}(A) \subseteq \sigma_{b}^{T}(A)$. If $z_{1} \in \sigma(B) \backslash \sigma_{e}(B)$, then $z_{1}$ is not an isolated point and hence, by Theorem $2,\left(z_{1}, z_{2}\right)$ is not an isolated point of $\sigma^{T}(A)$. This implies that $\left(z_{1}, z_{2}\right) \in \sigma_{b}^{T}(A)$, and hence 
$\sigma_{b}(B) \times \sigma(C) \subseteq \sigma_{b}^{T}(A)$. Similarly, one shows that $\sigma(B) \times \sigma_{b}(C) \subseteq \sigma_{b}^{T}(A)$.

Thus we have

$$
\left\{\sigma_{b}(B) \times \sigma(C)\right\} \cup\left\{\sigma(B) \times \sigma_{b}(C)\right\} \subseteq \sigma_{b}^{T}(A) .
$$

Conversely, suppose $z=\left(z_{1}, z_{2}\right) \in \sigma^{T}(A)$ but

$z \epsilon^{\prime}\left\{\sigma_{b}(B) \times \sigma(C)\right\} \cup\left\{\sigma(B) \times \sigma_{b}(C)\right\}$. Then $z_{1} \in \sigma(B) \backslash \sigma_{b}(B)$ and

$z_{2} \in \sigma(C) \backslash \sigma_{b}(C)$. Hence by Lemma 17 of [12] we obtain that $z \in \rho_{e}^{2}(A)$.

But $\rho_{e}^{2}(A) \subseteq \rho_{e}^{T}(A)$. Thus by Theorem 2 we conclude that $z$ is an isolated point of $\sigma^{T}(A)$ and hence $z \epsilon^{\prime} \sigma_{b}^{T}(A)$. This implies that

$\sigma_{b}^{T}(A) \subseteq\left\{\sigma_{b}(B) \times \sigma(C)\right\} \cup\left\{\sigma(B) \times \sigma_{b}(C)\right\}$. Thus

$$
\begin{aligned}
\left\{\sigma_{b}(B) \times \sigma(C)\right\} \cup\left\{\sigma(B) \times \sigma_{b}(C)\right\} \subseteq \sigma_{b}^{T}(A) & \subseteq \sigma_{b}^{1}(A) \subseteq \sigma_{b}^{2}(A) \\
& =\left\{\sigma_{b}(B) \times \sigma(C)\right\} \cup\left\{\sigma(B) \times \sigma_{b}(C)\right\} .
\end{aligned}
$$

This proves the theorem.

Thus the theorem illustrates the fact that analogous to joint spectra (Theorem 2) the various notions of joint Browder spectra $\left\{\sigma_{b}^{j}(A), j=1,2, T\right\}$ also coincide for these special class of operators on Hilbert spaces.

THEOREM 8. If $B$ and $C$ are, respectively, operators on Banach spaces $X_{1}$ and $X_{2}$ with $A_{1}=B \otimes 1, A_{2}=1 \otimes C$ and $A=\left(A_{1}, A_{2}\right)$, then

$$
\sigma_{b}^{1}(A)=\sigma_{b}^{2}(A)=\left\{\sigma_{b}(B) \times \sigma(C)\right\} \cup\left\{\sigma(B) \times \sigma_{b}(C)\right\}
$$

We leave the proof to the reader.

REMARK 3. Whether Theorems 7 and 8 are true for polynomial joint Browder spectrum is an open question. 


\section{References}

[1] W. Arveson, "Subalgebras of $C^{*}$-algebras II", Acta Math. 128 (1972), $271-308$.

[2] S.K. Berberian, "The Weyl spectrum of an operator", Indiana Univ. Math. J. 20 (1970), 529-544.

[3] F.E. Browder, "On the spectral theory of elliptic differential operators I", Math. Ann. 142 (1961), 22-130.

[4] J.J. Buoni, A.T. Dash and B.L. Wadhaw, "Joint Browder spectrum", Pacific J. Math. 94 (1981), 259-263.

[5] Z. Ceausescu and F.-H. Vasilescu, "Tensor products and Taylor's joint spectrum", Studia Math. 62 (1978), 305-31l.

[6] R.E. Curto, "Fredholm and invertible n-tuples of operators. The deformation problem", Trans. Amer. Math. Soc. 266 (1981), 129-159.

[7] A.T. Dash, "Joint spectra", Studia Math. 45 (1973), 225-237.

[8] A.T. Dash, "Joint essential spectra", Pacific J. Math. 64 (1976), 119-128.

[9] A.T. Dash, "On a conjecture concerning joint spectra", J. Funct. Anal. 6 (1970), 165-171.

[10] A.T. Dash and M. Schechter, "Tensor products and joint spectra", Israel J. Math. 8 (1970), 191-193.

[11] P.A. Filmore, J.G. Stampfli and J.P. Williams, "On the essential numerical range, the essential spectrum and a problem of Halmos", Acta Sci. Math. Szeged 33 (1972), 179-192.

[12] M. Schechter and M. Snow, "The Fredholm spectrum of tensor products", Proc. Roy. Irish Acad. Sect. A 75 (1975), 121-128.

[13] M. Snow, "A joint Browder essential spectrum", Proc. Roy. Irish Acad. Sect. A 75 (1975), 129-131.

[14] J.L. Taylor, "A joint spectrum for several commuting operators", $J$. Funct. Anal. 6 (1970), 172-191. 
[15] F.-H. Vasilescu, "A characterization of joint spectrum in Hilbert spaces", Rev. Roumaine Math. Pures Appl. 22 (1977), 1003-1009.

Department of Mathematics and Statistics,

College of Physical Science,

University of Guelph,

Guelph,

Ontario,

Canada NIG 2WI. 